\title{
Fatores de risco para a síndrome da apneia e hipopneia obstrutiva do sono em docentes de odontologia
}

\author{
Risk factors for obstructive sleep apnea/hypopnea syndrome in \\ dentistry professors
}

\author{
Carmem Dolores de Sá Catão* \\ Vicente Jadson Gregório Freitas ${ }^{* *}$ \\ Layanne Soyara Bidô Alves*** \\ Jackson Borba da Cruz ${ }^{* * *}$ \\ Ana Isabella Arruda Meira Ribeiro ${ }^{* * * *}$ \\ Maria Carolina Bandeira Macena*
}

\section{Resumo}

Introdução: a síndrome da apneia e hipopneia obstrutiva do sono (Sahos) é caracterizada pelo colapso e obstrução da via aérea superior durante o sono. Apresenta como sinais e sintomas ronco, sonolência excessiva e pausas respiratórias durante o sono. Trata-se de uma doença crônica, evolutiva, com alta taxa de morbidade e mortalidade, que pode levar ao comprometimento das atividades diurnas, expondo os portadores a risco de acidentes, além de estar associada às complicações cardiovasculares. Objetivo: verificar a prevalência de fatores de risco para a Sahos em professores de Odontologia, por meio do questionário sintomatológico, da escala de sonolência de Epworth (ESE) e das medidas antropométricas. Resultados: a amostra foi censitária, composta por 36 professores, $55,6 \%$ do sexo feminino e $69,4 \%$ do sexo masculino. Dos entrevistados, 30,5\% apresentaram pressão arterial (PA) superior a 120/80 $\mathrm{mmHg}, 52,8 \%$ encontravam-se acima do peso (índice de massa corporal - IMC > 25), 22,3\% apresentaram-se na escala de obesidade, 33,5\% exibiram circunferência do pescoço maior ou igual a $40 \mathrm{~cm}, 50 \%$ relataram roncar e $72,2 \%$ afirmaram despertar durante a noite. Apesar de 44,5\% afirmarem ter um sono agitado, apenas 5,6\% observaram ou foram avisados de que paravam de respirar enquanto dormiam. Conclusões: pôde-se observar um alto percentual de docentes com grau excessivo de sonolência por meio da $\operatorname{ESE}(61,1 \%)$, ape- sar da ausência de relação estatisticamente significativa com as variáveis da pesquisa. O IMC aumentado, níveis elevados de PA (>140/90 $\mathrm{mmHg}$ ), ronco e sono agitado foram sintomas encontrados na população estudada, evidenciando um elevado risco para o desenvolvimento da Sahos.

Palavras-chave: Síndrome da apneia do sono. Distúrbios do sono por sonolência excessiva. Ronco. Docentes de Odontologia.

\section{Introdução}

Muito do que se sabe a respeito dos distúrbios respiratórios do sono foi descoberto em 1965, quando pesquisadores observaram apneias durante o sono, decorrentes da obstrução de vias aéreas superiores, em pacientes com síndrome de Pickwick, como era conhecida a síndrome da apneia e hipopneia do sono (Sahos) ao ser primeiramente descrita ${ }^{1}$.

Considerada um problema de saúde pública, a Sahos aumenta a morbimortalidade cardiovascular e os acidentes de trânsito ${ }^{2}$ e caracteriza-se por episódios recorrentes de obstrução parcial ou completa das vias aéreas superiores durante o sono. O fluxo

Professora doutora, Departamento de Medicina da Universidade Federal de Campina Grande, Campina Grande, PB, Brasil. Graduando em Odontologia, Departamento de Odontologia da Universidade Federal de Campina Grande, Patos, PB, Brasil. Graduanda em Odontologia, Departamento de Odontologia da Universidade Federal de Campina Grande, Patos, PB, Brasil.

Professor mestre, Departamento de Medicina da Universidade Federal de Campina Grande, Campina Grande, PB, Brasil.

Professora doutora, Departamento de Odontologia da Universidade Estadual da Paraíba, Campina Grande, PB, Brasil.

Professora doutora, Departamento de Odontologia da Universidade Federal de Campina Grande, Patos, PB, Brasil. 
aéreo é diminuído na hipopneia ou completamente interrompido na apneia, a despeito do esforço respiratório ${ }^{3}$. Apresenta como sinais e sintomas o ronco, a interrupção da respiração de forma intermitente durante o sono, levando ou não a interrupção do sono, agitação ao dormir, sensação de sufocamento ao despertar, sonolência excessiva diurna, por conta da má qualidade do sono, impotência sexual, cefaleia, irritabilidade, depressão e ansiedade ${ }^{4,5}$.

Os fatores associados à Sahos são hipertensão arterial sistêmica (HAS), hipertensão pulmonar, arritmias cardíacas relacionadas ao sono, à angina noturna, ao refluxo gastresofágico, ao prejuízo da qualidade de vida e insônia ${ }^{6}$. Entre os fatores predisponentes estão: histórico familiar, obesidade, aumento da circunferência do pescoço, e da relação cintura-quadril, hipotireoidismo, acromegalia, obstrução nasal, diabetes, insuficiência renal crônica e gravide $z^{7}$, bem como índice de massa corporal aumentado, roncos habituais, sono não reparador, fadiga, idade acima de 40 anos e sexo masculino. As estimativas indicam que a relação homem/mulher varia entre $2: 1$ e $4: 1^{8,9}$.

Embora a relação entre obesidade e apneia obstrutiva do sono e ronco não seja completamente entendida, parece que a obesidade comprime a faringe e aumenta sua colapsabilidade. $\mathrm{O}$ aumento na circunferência do pescoço, com valores superiores a $35 \mathrm{~cm}$ para mulher e $40 \mathrm{~cm}$ para homens, é um dos marcadores para deposição central de gordura e tem demonstrado ser importante preditor de ronco e apneia obstrutiva do sono ${ }^{10,11}$. A deposição aumentada de gordura ou de tecido mole na região do pescoço, que ocorre principalmente em obesos com IMC superior a 30, ou adjacente às vias aéreas superiores (VAS), causa uma possível constrição, que é apontada como responsável pela apneia em obesos ${ }^{12,13}$.

Algumas características anatômicas também podem colaborar para o diagnóstico, como: presença de retrognatismo, palato mole aumentado, hipertrofia tonsilar e macroglossia ${ }^{14}$. Além da análise clínica, existem outros métodos utilizados para a investigação dos distúrbios do sono, que vão desde avaliações subjetivas, por meio da aplicação de questionários específicos, aos registros polissonográficos diurnos ou noturnos. Os questionários, em sua maioria, são internacionais e passam por validação após sua tradução para a língua portuguesa. Entre eles podemos citar o questionário da escala de sonolência de Epworth (ESE) que tem uma pontuação de 0 a 24, caracterizando a sonolência excessiva para valores a partir de $10^{15}$.

A terapêutica da Sahos tem caráter multidisciplinar e engloba desde medidas clínicas, em que um dos principais tratamentos é realizado com o uso de pressão aérea positiva contínua, com o auxílio de um aparelho que direciona um fluxo contínuo de ar através de um tubo para uma máscara instalada no paciente, além de redução do peso, uso de antidepressivos, estimulação elétrica, até cirúrgicas, fazendo-se necessária a participação do cirurgião-dentista, seja por meio do emprego de aparelhos intrabucais ou na realização de cirurgias bucomaxilares. O papel do cirurgião-dentista é construir aparelhos intrabucais de uso noturno, capaz de desobstruir a passagem do ar, permitindo melhor ventilação do paciente, e o controle dos efeitos secundários e colaterais nas estruturas bucofaciais, que são mais eficientes em casos leves e moderados, nas classes II e III de Fujita, nas quais o paciente não apresenta disfunção temporomandibular ${ }^{9,16,17}$.

Diante do exposto, a presente pesquisa visou verificar a prevalência de fatores de risco para a Sahos em professores de Odontologia de uma universidade pública da Paraíba, além de enfatizar a importância da qualidade do sono e suas consequências, com o objetivo de auxiliar no diagnóstico de possíveis distúrbios do sono a fim de garantir melhor qualidade de vida.

\section{Materiais e método}

O presente artigo trata de uma pesquisa quantitativa, de caráter transversal descritivo, desenvolvida com os docentes do curso de Odontologia da Universidade Federal de Campina Grande (UFCG), em plena atividade na instituição. Caracteriza-se pela quantificação das modalidades de coleta de informações, bem como pela identificação de sinais e de sintomas, por meio da escala de sonolência de Epworth $^{18}$, do questionário sobre a sintomatologia da Sahos ${ }^{19}$ e da coleta das medidas antropométricas e de pressão arterial.

A pesquisa foi realizada com uma população de 38 professores, no horário de trabalho, após a leitura e assinatura do termo de consentimento livre e esclarecido, responderam aos questionários, 36 canditados, correspondendo a aproximadamente 95\% do universo amostral. O trabalho foi devidamente aprovado pelo Comitê de Ética em Pesquisa em Seres Humanos do Hospital Universitário Alcides Carneiro), de acordo com o Parecer n⿳ํㅜ 386.302. Os critérios de exclusão foram: estar afastado por licença médica ou maternidade, não ser professor do curso de Odontologia, se recusar em responder o questionário.

O questionário foi aplicado por um aluno do curso de Odontologia da UFCG para avaliar condições subjetivas relacionadas à Sahos bem como verificar possíveis distúrbios do sono. Constava no instrumento de coleta de dados um questionário geral com os dados sociodemográficos, as medidas antropométricas (altura e peso) - aferidas com uma fita métrica e uma balança calibrada e certificada pelo Inmetro-, a medida da circunferência do pescoço - também verificada com fita métrica-, o valor do índice de massa corpórea e a pressão arterial. Na verificação da pressão arterial, o professor encontrava-se sentado, após relaxar por 5 minutos com o braço apoiado sobre 
uma bancada, aproximadamente na altura do peito, para tanto se utilizou um esfigmomanômetro e estetoscópio certificados pelo Inmetro. Houve ainda a coleta de dados relacionados à sintomatologia da Sahos, além dos da escala de sonolência de Epworth.

Os dados obtidos por intermédio da ESE foram autoadministrados, e os indivíduos foram solicitados a graduar, em uma escala do tipo Likert, de zero a três, qual a chance de adormecer em oito situações, baseados na sua vida habitual nos últimos tempos, feita uma distinção entre dormir e simplesmente sentir-se cansado. Se o indivíduo não experimentou nenhuma das situações propostas recentemente, pede-se que, mesmo assim, ele estime a chance de dormir. O escore total pode variar de zero a 24. A ESE mostra-se capaz de distinguir indivíduos e grupos diagnósticos com um amplo espectro de sonolência diurna ${ }^{18}$.

Os entrevistados que apresentaram o somatório total na ESE entre zero a cinco pontos foram encaixados nas seguintes classificações: ótima (boa noite de sono); de seis a oitopontos, como atenção para outros sinais de apneia (ronco, sonolência); de nove a 24 pontos a sonolência excessiva pode ser devida à apneia.

É importante ressaltar que o questionário sobre a sintomatologia da Sahos ${ }^{19}$ tem caráter complementar, auxiliando a escala de Epworth em um diagnóstico mais preciso.

As etapas de processamento, armazenamento e análise dos dados foram realizadas com o programa estatístico SPSS $18.0^{\circledR}$ (Statistical Package for the Social Sciences) (SPSS Inc., Chicago, Illinois, USA) e analisados por meio de estatística descritiva e inferencial uni e bivariada. Para a interpretação das informações, foi adotado um intervalo de confiança (IC) de 95\% e nível de significância de $5 \%(\mathrm{p}<0,05)$.

\section{Resultados}

O universo da pesquisa foi composto por 38 professores, os critérios de exclusão foram aplicados em dois casos de licença-maternidade, correspondendo a 5,3\% da população. Após a análise estatística, constatou-se que houve um predomínio de professores do sexo feminino, $55,6 \%$ dos questionários tidos como válidos, e $69,4 \%$ casados.

Com relação às medidas de pressão arterial (PA), 69,5\% apresentaram valor menor ou igual a $120 / 80 \mathrm{mmHg}$, e $30,5 \%$ encontravam-se acima desse valor.

Grande parcela dos docentes, 33,5\%, apresentou a circunferência do pescoço maior ou igual a $40 \mathrm{~cm}$. Dos homens, $60 \%$ apresentaram circunferência do pescoço maior que $40 \mathrm{~cm}$, e entre as mulheres, aproximadamente $40 \%$ têm valores superiores a $35 \mathrm{~cm}$.

Dos entrevistados, $30,6 \%$ estavam acima do peso, e 22,3\% apresentavam algum grau de obesidade (Tabela 1). Os professores do sexo masculino apresentaram maior IMC, evidenciando uma relação estatisticamente significante $(p=0,002)$ entre as duas variáveis.
Tabela 1 - Índice de massa corpórea dos professores investigados (Patos, PB, Brasil, 2014)

\begin{tabular}{l|r}
\multicolumn{1}{c|}{ IMC } & Frequência / \% \\
\hline Peso normal & $17(47,2 \%)$ \\
Acima do peso & $11(30,6)$ \\
Obesidade 1 & $6(16,7 \%)$ \\
Obesidade 2 & $1(2,8)$ \\
Obesidade 3 & $1(2,8)$ \\
Total & $36(100 \%)$ \\
\hline
\end{tabular}

Fonte: elaboração dos autores com dados da pesquisa.

Quanto aos achados subjetivos, quando questionados sobre acordar durante a noite, $72,2 \%$ dos sujeitos relataram que acordavam durante a noite, desses, $19,2 \%$, declararam que com frequência, já $50 \%$ dos entrevistados foram informados que roncam ao dormir.

Apesar de 44,5\% dos investigados ter respondido que apresentam sono agitado, apenas $5,6 \%$ disseram que já observaram, ou foram avisados, de que paravam de respirar enquanto dormem. $\mathrm{Na}$ análise dos dados, $75 \%$ apresentaram cansaço excessivo, desses, 33,3\% relataram que grande frequência. Assim como situações de sonolência diurna, pois $77,8 \%$ apresentaram essa característica. Dos entrevistados, 5,6\% já sofreram algum acidente de trânsito por conta da sonolência excessiva. A facilidade em ficar irritado foi uma das perguntas, e 50\% afirmaram essa ocorrência ultimamente.

Quando indagados sobre o uso de álcool, $75 \%$ afirmaram beber apenas nos finais de semana; sobre o uso de ansiolíticos, 13,9\% utilizavam esses medicamentos, sobre o fato de ser fumante apenas $8,3 \%$ responderam afirmativamente.

Ao aplicar a escala de sonolência de Epworth, observou-se que $27,8 \%$ dos professores têm boa noite de sono, $11,1 \%$ devem atentar para outros sinais da apneia e $61,1 \%$ apresentam sonolência excessiva, o que torna esse resultado preocupante (Tabela 2).

Tabela 2 - Dados da escala de sonolência de Epworth aplicada aos professores investigados (Patos, PB, Brasil, 2014)

\begin{tabular}{l|l}
\hline \multicolumn{1}{c|}{ ESE } & \multicolumn{1}{c}{ Frequência / \% } \\
\hline Boa noite de sono & $10(27,8 \%)$ \\
Atenção para outros sinais de apneia & $4(11,1 \%)$ \\
Sonolência excessiva & $22(61,1 \%)$ \\
Total & $36(100 \%)$ \\
\hline
\end{tabular}

Fonte: elaboração dos autores com dados da pesquisa.

\section{Discussão}

A efetividade da análise dos questionários sintomáticos para diagnóstico da Sahos é comprovada pelo estudo de Noal et al. ${ }^{20}$ (2008), os quais, em pesquisa com 3.136 habitantes de Pelotas, constataram que $50,5 \%$ dos entrevistados confessaram roncar, desses, $9,9 \%$ eram portadores da Sahos, corroboran- 
do os achados desta pesquisa. Apesar de o sexo masculino ser um fator de risco para a síndrome, como mostra o estudo de Pedrosa et al..$^{21}$ (2008), nesta pesquisa, a maioria dos entrevistados era do sexo feminino e, mesmo assim, o percentual de docentes com sonolência excessiva foi elevado, passando dos $61 \%$.

$\mathrm{O}$ aumento na circunferência do pescoço tem demonstrado ser importante preditor de ronco e apneia obstrutiva do sono ${ }^{10,11}$. Os entrevistados apresentavam, em sua maioria, acima da média em relação às medidas esperadas para a circunferência do pescoço $(>40 \mathrm{~cm})$ para cada gênero ${ }^{22}$. Assim como a circunferência do pescoço, o grande número de obesos revelou-se um dado preocupante, já que o IMC é também um fator predisponente para a Sahos ${ }^{7}$.

O percentual de educadores com pressão arterial alta superou os dados de prevalência nacional de hipertensão autorreferida, visto que chegou a $21,6 \%$ da população em estudo, um dado preocupante, já que a pressão arterial acima do normal pode ser um fator predisponente à $\mathrm{Sahos}^{8,23}$.

$\mathrm{O}$ cansaço e a sonolência excessivos durante o dia podem ser sinais e/ou sintomas da $\mathrm{Sahos}^{24}$. A jornada de trabalho e as condições a que os professores são submetidos na sua profissão podem gerar repercussões em seu estado de saúde. Relacionando esses preceitos com o tema deste trabalho, observou-se que a maior parte dos entrevistados apresentou sonolência excessiva, podendo ser justificada pela rotina de trabalho dos professores do curso de Odontologia e suas atividades laboratoriais, além de outras atividades de trabalho, levando a uma sobrecarga. Assim como no estudo de Costa et al. ${ }^{25}$ (2012), em que 4\% dos entrevistados, classificados no escore sonolência excessiva, declararam ter cansaço excessivo, sono agitado e parada de respiração durante a noite. Isso conduz a uma indicação para realização de polissonografia para confirmação da existência da Sahos.

Os fatores anatômicos avaliados pelas medidas antropométricas, como o IMC e CP, a pressão arterial acima do desejável e a sonolência excessiva podem causar déficit de memória e de atenção, diminuindo o rendimento no trabalho ${ }^{26}$.

O uso de ansiolíticos, fumo e álcool, pelos professores foi consideravelmente baixo. O uso de álcool não é frequente, assim, não apresentou relação com a ocorrência de sinais e sintomas da síndrome nessa população.

O questionário sobre a sintomatologia da Sahos tem caráter complementar, auxiliando a escala de Epworth, para um diagnóstico mais preciso ${ }^{19}$. Desse modo, o diagnóstico precoce é extremamente necessário, pois essa síndrome é um fator agravante de diversas patologias com alto grau de mortalidade, como as complicações cardiovasculares ${ }^{27,28}$.

\section{Conclusão}

Pôde-se observar um alto percentual de sujeitos com grau excessivo de sonolência, verificados por intermédio da escala de sonolência de Epworth, apesar de não ter apresentado uma relação estatisticamente significativa com as variáveis da pesquisa, contudo, corrobora com os dados obtidos pelo questionário sintomatológico. O IMC aumentado, níveis elevados de PA, ronco e sono agitado foram encontrados na população estudada, evidenciando um elevado risco para o desenvolvimento da Sahos.

Os resultados deste estudo podem ser aplicados em outras populações de professores, visto que as condições de trabalho são inadequadas, o que pode causar a confusão de diagnóstico da Sahos nos professores sujeitos à carga excessiva de trabalho e déficit na qualidade do sono. Julga-se, assim, necessária a indicação de polissonografia para alguns dos professores, para possível diagnóstico e tratamento da síndrome.

\section{Abstract}

Introduction: The syndrome of apnea and obstructive sleep hypopnea syndrome (Sahos) is characterized by the collapse and obstruction of the upper airway during sleep. It presents as signs and symptoms, snoring, excessive sleepiness and breathing pauses during sleep. It is a chronic, progressive, with high morbidity and mortality, which can lead to impairment of daytime activities, exposing patients to risk of accidents, as well as being associated with cardiovascular complications. Objective: To determine the prevalence of risk factors for OSAS in Dentistry teachers, through symptomatic questionnaire, the Epworth Sleepiness Scale (ESS) and anthropometric measures. Results: The sample census, made up of 36 teachers, $55.6 \%$ female and $69.4 \%$. Of the respondents, $30.5 \%$ had blood pressure $(B P)$ greater than 120/80 $\mathrm{mmHg}, 52.8 \%$ were overweight (body mass index - BMI> 25), 22.3\% presented themselves on the scale obesity, $33.5 \%$ exhibited neck circumference greater than or equal to $40 \mathrm{~cm}, 50 \%$ reported snoring and $72.2 \%$ said they awaken during the night. Although $44.5 \%$ claiming to have a restless sleep, only $5.6 \%$ observed or were warned that stopped breathing while asleep. Conclusions: it was observed a high percentage of teachers with excessive degree of sleepiness by ESS $(61.1 \%)$, despite the absence of statistically significant relationship with the variables of the research. The increased BMI, high blood pressure levels (> 140/90 $\mathrm{mmHg}$ ), snoring and restless sleep were found in the population studied, indicating a high risk for the development of Sahos.

Keywords: Sleep apnea syndromes. Disorders of excessive somnolence. Snoring. Dentistry Professors. 


\section{Referências}

1. Gastaut H. Polygraphic study of episodic diurnal and nocturnal manifestation of the pickwickian syndrome. Behav Brain Res 1965; 2:167-8.

2. Boari L, Cavalcanti CM, Bannwart SRFD, Sofia OB, Dolci JEL. Avaliação da escala de Epworth em pacientes com a síndrome da apneia e hipopneia obstrutiva do sono. Rev Bras Otorrinolaringol 2004; 70(6):752-6.

3. Martins AB, Tufik S, Moura SMGPT. Síndrome da apneia-hipopneia obstrutiva do sono. Fisiopatologia. J Bras Pneumol 2007; 33(1):93-100.

4. Cavallari FEM, Leite MGJ, Mestriner PRE, Couto LGF, Fomin DS, Oliveira JAA. Relação entre hipertensão arterial sistêmica e síndrome da apneia obstrutiva do sono. Rev Bras Otorrinolaringol 2002; 68(5):619-22.

5. Valera FCP, Demarco RC, Lima WTA. Síndrome da Apneia e da hipopneia obstrutivas do sono (Sahos) em crianças. Rev Bras Otorrinolaringol 2004; 70(2):232-7.

6. American Academy of Sleep Medicine Task Force (AASM). Sleeprelated breathing disorders in adults: recommendations for syndrome definition and measurement techniques in clinical research. Sleep Science 1999; 22(5):667-89.

7. Drager LF, Ladeira RT, Brandão-Neto RA, Lorenzi-Filho G. Apneia do sono e risco cardiovascular. Rev Factores de Risco $2010 ; 17: 20-6$

8. Bixler EO, Vgontzas AN, Lin H, Have TT, Rein J, VelaBueno A. Prevalence of sleep disorder breathing in womeneffects of gender. Am J Respir Crit Care Med 2001; 163(3 Pt1):608-13.

9. Flemons WW, Douglas NJ, Kuna ST, Rodenstein DO, Wheatley, J. Access to diagnosis and treatment of patients with suspected sleep apnea. Am J Respir Crit Care Med 2004; 169(6):668-72.

10. Young T, Palta M, Dempsey J, Skatrud J, Weber S, Badr S. The occurrence of sleep disordered breathing among middle-aged adults. N Engl J Med 1993. 328(17):1230-5.

11. Davies RJ, Ali NJ, Stradling JR. Neck circumference and other clinical features in the diagnosis of the obstructive sleep apnea syndrome. Thorax 1992; 47(2):101-5.

12. Schafer H, Pauleit D, Sudhop T, Gouni-Berthold I, Ewig S, Berthold HK. Body fat distribution, serum leptin, and cardiovascular risk factors in men with obstructive sleep apnea. Chest 2002; 122(3):829-39.

13. Ip MS, Lam KS, Ho C, Tsang KW, Lam W. Serum leptin and vascular risk factors in obstructive sleep apnea. Chest 2000; 118(3):580-6.

14. Schwab RJ, Pasirstein M, Pierson R, Mackley A, Arens R, Maislin G. Anatomic risk factors for sleep apnea. Am J Respir Crit Care Med 2003; 168:522-30.

15. Togeiro SMGP, Smith AK. Métodos diagnósticos nos distúrbios do sono. Rev Bras Psiquiatr 2005; 27 Suppl I:8-15.

16. Ito, FA, Ito RT, Moraes NM, Sakima T, Bezerra MLS, Meirelles $\mathrm{R}$ C. Condutas terapêuticas para tratamento da síndrome da Apneia e Hipopneia obstrutiva do dono (Sahos) e da díndrome da resistência das vias aéreas superiores (SRVAS) com enfoque no aparelho antirronco (AAR-ITO). R Dental Press Ortodon Ortop Facial, 2005; 10(4):143-56.

17. Araujo LG, Coelho PR, Guimarães JP. Tratamento da síndrome de apneia-hipopneia obstrutiva do sono por meio de placa protrusiva mandibular. Rev Fac Odontol Univ Passo Fundo 2011; 16(1):100-4

18. Johns MW. A new method for measuring daytime sleepiness: The Epworth Sleepiness Scale. Sleep Science 1991; 14(6):540-5
19. Musman S. Avaliação de modelo de predição para apneia do sono em pacientes submetidos à polissonografia [Dissertação de Mestrado]. Belo Horizonte: Faculdade de Medicina, Universidade Federal de Minas Gerais; 2008.

20. Noal RB, Menezes AMB, Canani SF. Ronco habitual e apneia obstrutiva observada em adultos: estudo de base populacional. Rev Saude Publica 2008; 42(2):224-33.

21. Pedrosa RP, Lorenzi-Filho G, Drager LF. Síndrome da apneia obstrutiva do sono e doença cardiovascular. Rev Med 2008; 87(2):121-7.

22. Martin SE, Mathur R, Marshall I, Douglas NJ. The effect of age, sex, obesity and posture on upper airway size. Eur Respir J 1997; 10:2087-90.

23. Schmidt MI, Duncan BB, Hoffmann FF, Moura L, Malta DC, Carvalho RMSV. Prevalência de diabetes e hipertensão no Brasil baseada em inquérito de morbidade autorreferida, Brasil, 2006. Rev Saude Publica. 2009; 43 Suppl 2:74-82.

24. Bittencourt LRA, Haddad FM, Fabbro CD, Cintra FD, Rios L. Abordagem geral do paciente com síndrome da apneia obstrutiva do sono. Rev Bras Hipertens 2009; 16(3):158-63.

25. Costa RO, Farias ABL, Ribeiro AIAM, Catão MHCV, Costa IRRS, Catão CDS. Escala de sonolência de Epworth detecta sintomas da apneia do sono em docentes de Odontologia. Rev Bras Odontol 2012; 69(2):228-31.

26. Mancini MC, Aloe F, Tavares S. Apneia do sono em obesos. Arq Bras Endocrinol Metab 2000; 44(1):81-90

27. Godolfim LR. Apneia obstrutiva do sono. J Bras Ortodon Ortop Facial 2006; 11(65/66):484-501.

28. Cintra FD, Poyares D, Guilleminault C, Carvalho AC, Tufik S, Paola AAV. Alterações cardiovasculares na síndrome da apneia obstrutiva do sono. Arq Bras Cardiol 2006; 86(6):399-407.

Endereço para correspondência:

Carmem Dolores de Sá Catão

Universidade Federal de Campina Grande Unidade Acadêmica de Ciências e da Saúde Juvêncio Arruda, 795, Bodocongó 58407-485 - Campina Grande, Paraíba, Brasi Telefones: 558321011239 / 558388831110

E-mail: cataocarmem@gmail.com

Recebido: 24/02/15. Aceito: 10/09/15. 